\title{
Field-Deployable Raman Anti-Counterfeit Screening of Tamiflu Capsules
}

\author{
Yvette L. Loethen, Jason D. Rodriguez \\ US Food and Drug Administration, Center for Drug Evaluation and Research, Division of Pharmaceutical \\ Analysis, St. Louis, USA \\ Email: Jason.Rodriguez@fda.hhs.gov
}

Received 31 March 2015; accepted 18 May 2015; published 20 May 2015

Copyright (C) 2015 by authors and Scientific Research Publishing Inc.

This work is licensed under the Creative Commons Attribution International License (CC BY). http://creativecommons.org/licenses/by/4.0/

(c) (i) Open Access

\begin{abstract}
In an effort to promote the availability of safe and effective drugs, the US Food and Drug Administration is developing spectroscopic methods to assess the quality of drugs in the field. Here we report a rapid screening classification method for Tamiflu (oseltamivir phosphate) capsules using a portable Raman spectrometer to perform screening on three solid oral dosage strengths of Tamiflu, $30 \mathrm{mg}, 45 \mathrm{mg}$ and $75 \mathrm{mg}$. Tamiflu is an antiviral drug that is stockpiled for use in the event of pandemic influenza outbreak. The qualitative classification methods reported were developed using the Raman spectra of intact capsules. The classification algorithms used were able to reliably distinguish the three dosage strengths of Tamiflu. These qualitative models are validated with additional Tamiflu samples from different batches and simulated counterfeits of Tamiflu. The probability that a test sample belongs to each dosage strength class is calculated, and strict class predictions are used to assign each sample to a particular class. The classification methods reported here enable development of user-independent, field-deployable methods for finished drug products and are able to correctly assign $92 \%$ of the validation samples using authentic Tamiflu and $100 \%$ of the simulated counterfeits.
\end{abstract}

\section{Keywords}

Anti-Counterfeit, Portable Instruments, Raman Screening

\section{Introduction}

Antivirals are considered priority medicines since they play an important role in controlling pandemic outbreaks and are stockpiled by governments across the world [1]. The development of rapid screening tools to assure that stockpiled medicines are authentic is important in light of recent reports of infiltration of counterfeits in the antibiotic and antiviral supply chain [2]. Unlike vaccines that require yearly updating to match the expected strain 
of seasonal influenza, antiviral drugs—particularly solid oral dosage forms—can be stockpiled by governments for use in the event of an outbreak years in advance. Of the antiviral drugs available to combat influenza, Tamiflu is the most common. As of 2009, Tamiflu comprised 40 million of the 50 million regimens available in the strategic national stockpile [1]. The outbreaks of swine influenza A (H1N1) in 2009 highlighted the importance of emergency preparedness in the event of pandemic influenza. Tamiflu was at the center of the US action plan for treatment and prophylaxis of the swine flu. The US Food and Drug Administration (FDA) issued an emergency use authorization [3] to allow for use in pediatric patients and to streamline distribution of the medication by federal, state and local jurisdictions, including releasing regimens from the federal government's stockpile [4]. Over the past decade [5], there have been several instances where Tamiflu counterfeits have appeared across the globe. Tamiflu counterfeits have been found to contain antibiotic and analgesics instead of the approved active ingredient, oseltamivir phosphate.

Over the past few years, portable Raman spectrometers have allowed screening of pharmaceutical materials to expand outside the laboratory and into the field [6]. These units give investigators the flexibility of making realtime measurements to screen drug products and are particularly useful when coupled with multivariate methods. Methods, such as principal component analysis (PCA) and soft independent modelling of class analogies (SIMCA), are commonly used to detect counterfeit products [7]. Despite the studies [8] detailing successful implementation of portable Raman instruments to screen tablet-form drug products, the number of studies including capsule-form products is conspicuously lagging. This is likely due to the inherent difficulties in measurement of capsules (primarily colored ones) by Raman. Capsules typically give rise to a strong fluorescence signal, which overwhelms the Raman signal of the internal capsule contents. Fluorescence makes robust method development challenging and has limited analysis of capsule-form products to being performed using high resolution spectrometers or specialized Raman set-ups such as wide angle illumination or transmission Raman [9], which are not readily deployed in the field. Capsule-form products may be screened by emptying the capsules and analyzing the composites; however, since these methods are designed for field use, procedures requiring little to no sample preparation are preferred. By measuring intact capsules, we are also able to preserve the non-destructive, non-invasive nature of Raman spectroscopy since a major goal of our field program is to preserve the sample in the case further testing may be necessary.

In this paper, we report on the development of Raman screening methods by which the safety and efficacy of priority drugs such as Tamiflu can be evaluated at point of use or in stockpiles. A commercially-available portable Raman spectrometer is used in this proof of concept to develop screening methods for intact capsules of Tamiflu at three common dosage strengths of Tamiflu: $30 \mathrm{mg}, 45 \mathrm{mg}$ and $75 \mathrm{mg}$. Exploratory studies were initially performed using PCA methods to confirm that Raman could be used to distinguish between the different dosages strengths. A SIMCA classification model was then created for each of the three dosage strengths and method validation and robustness testing was performed for both PCA and SIMCA models using different batches of the three dosage strengths and simulated counterfeit samples. The simulated counterfeits included samples of other capsule form drug products-amantadine and acyclovir-and authentic Tamiflu capsules shells which were refilled with different contents. The contents of the re-filled simulated counterfeits were made to challenge the methods by mimicking what might be present in a counterfeit sample and appear to be physically and visually similar to the authentic product.

\section{Materials and Methods}

All materials were purchased and used as received. Authentic Tamiflu was obtained as capsules in three dosage strengths: 75 mg (NDC 0004-0800-85), 45 mg (NDC 0004-0801-85), and 30 mg (NDC 0004-0802-85). The calibration set used for method development contained 36 total samples-12 per dosage strength which were four capsules from three different boxes. The validation set consisted of 12 authentic Tamiflu samples-four capsules for each dosage strength, using a different box of Tamiflu (and different batches where available) from those used in the calibration set and the 20 simulated counterfeit samples including authentic Tamiflu capsule shells that had been refilled with different contents and amantadine and acyclovir capsules which are common antiviral drugs and the former is also used to treat influenza. The contents used for re-filling were starch, titanium dioxide, and acetaminophen mixtures. Three empty capsule shells, one for each dosage strength, were also tested as simulated counterfeits.

Raman spectra of drug products were acquired using a portable Raman instrument (B \& W Tek MiniRam II 
portable Raman spectrometer) with $785 \mathrm{~nm}$ excitation and $\sim 3 \mathrm{~mm}$ defocused spot size, using a custom tablet holder accessory to allow defocusing. The portable Raman instrument features a $180^{\circ}$ backscattering configuration. This type of configuration is common in commercially-available portable/handheld instruments. The excitation laser spot size is de-focused to accommodate a $3 \mathrm{~mm}$ sampling area which serves a dual purpose to decrease the localized laser fluence on the capsule and avoid burning colored capsules and increasing the area on and within the capsule which is analyzed by Raman. In order to make up for lower signal due to the de-focused laser spot, longer accumulation times are needed than would be required for the experiments using the normal nominal spot size of $\sim 180$ microns. Total accumulation times for Tamiflu capsules were 35 seconds for the lighter yellow-colored half of $30 \mathrm{mg}$ dosage, 30 seconds for the darker yellow-colored half of $30 \mathrm{mg}$ dosage, 50 seconds for each half of $45 \mathrm{mg}$ dosage and white half of $75 \mathrm{mg}$ dosage, and 40 seconds for yellow half of $75 \mathrm{mg}$ dosage. Total accumulation times varied for the simulated counterfeits from 15 to 50 seconds, using the same integration parameters as for Tamiflu capsules where possible. To build adequate variability in the model, nine spectra of each half of the capsule were acquired, with sample reposition and randomization in the laser spot location on the sample for each spectral acquisition being performed. Randomization is important when analyzing capsule products because the sides of capsules are generally indistinguishable. The resulting 18 spectra per capsule were averaged to yield 1 average spectrum per capsule. This approach was used to build as much variability as possible into the classification method and not make the method dependent on a particular orientation of the capsule. The other drug products had total accumulation times of $50 \mathrm{~s}$ for amantadine product and $15 \mathrm{~s}$ for acyclovir product (matching signal intensity since detector saturates at the integration times used for Tamiflu). Nine spectra were acquired and averaged for each of these drug products. Three spectra were acquired for pure oseltamivir phosphate, the active pharmaceutical ingredient (API) in Tamiflu, with an integration time of $50 \mathrm{~s}$ and averaged.

After spectral acquisition of the calibration set, some capsules for each dosage strength were emptied, refilled to at least half full with pregelatinized starch, inverted three times, and then emptied. This "starch rinse” was performed three times for each capsule. One capsule per dosage was set aside as an "empty capsule” sample. For preparation of simulated counterfeit samples, starch-rinsed capsules were then filled with appropriate materials. Re-filling materials included pregelatinized starch, titanium dioxide (rutile, 99.5\%, Alfa Aesar), and three binary mixtures of acetaminophen with pregelatinized starch, titanium dioxide, and talc (Fisher Scientific, laboratory grade powder). Binary mixtures were prepared as 50/50 mixtures by mass and milled for 10 minutes in powder mill (SPEX Sample Prep, model 5100 mixer/mill). Capsules were refilled such that the final mass of the capsule was similar to authentic Tamiflu samples (within +/- $0.005 \mathrm{~g}$ of average mass for calibration set). Due to differences in density across the materials, the capsules were difficult to fill sufficiently to achieve the desired final mass for some mixtures. In these cases the final masses were $+/-0.026 \mathrm{~g}$ of average mass for starch/acetaminophen and talc/acetaminophen binary mixtures. The other capsule-form drug products studied contained the active ingredients amantadine (100 mg, gel capsule, NDC 0378-2200-01) and acyclovir (200 mg, capsule, NDC 0527-1704-01). The pure active ingredient of Tamiflu, oseltamivir phosphate, was purchased from TSZ Chem and scanned in $4 \mathrm{~mL}$ glass vial.

Method development was performed using PLS Toolbox (Eigenvector Research, Inc. Version 7.9). The spectral region was restricted from 300 to $1850 \mathrm{~cm}^{-1}$ since most Raman features were in this range. Data preprocessing included first derivatization (Savitzky-Golay, second order polynomial, filter width of 31), multiplicative scattering correction (using the mean), and mean-centering. The use of first derivative preprocessing was used to minimize the spectral baseline due to fluorescence of the capsule signatures. Cross validation (venetian blinds, 6 data splits) was used to assist in retaining the appropriate amount of PCs for each model. The spectra were then analyzed separately by PCA and SIMCA. PCA was performed on the entire calibration set, encompassing all three dosages. Three PCs were chosen for the model which was then applied to the validation samples, simulated counterfeits and other drug products. The numbers of PCs chosen for the model were based on careful comparison between the principal component distributions and examination of the root mean squared error of cross validation (RMSECV) trends. In general, the number of PCs retained correspond to a minimum (or near minimum) RMSECV value.

SIMCA analysis was performed by assigning each spectrum in the calibration set to one of the three classes based on the three dosage strengths included in this study. An independent PCA model was created for each dosage strength. Two PCs were chosen each for the 75 and $45 \mathrm{mg}$ dosage strengths and three PCs were chosen for the $30 \mathrm{mg}$ capsules, based on comparison of the PCA distribution and RMSECV values as described above. The 
validation set and simulated counterfeit samples were then tested against the SIMCA model and a class prediction for each validation sample was made based on the probability of belonging to each of the three classes. Then the SIMCA algorithm compared spectra of validation samples to each class in the model and calculated a probability that the validation sample would be part of each class. The calculation of probability was performed by PLS Toolbox and has been described in detail elsewhere [10]. Briefly, the probability is determined using the "combined" decision rule based on the $\mathrm{Q}$ and $\mathrm{T}^{2}$ outlier statistics for each validation sample compared to $\mathrm{Q}$ and $\mathrm{T}^{2}$ distributions for each class. Each test sample may be assigned to only one class. If a sample was found to have low probability of belonging to any of the classes-or found to have high probability of belonging to multiple classes then no class designation is made.

\section{Results and Discussion}

The Raman spectra of the $30 \mathrm{mg}, 45 \mathrm{mg}$, and $75 \mathrm{mg}$ authentic Tamiflu capsules are shown in Figure 1(a), along with the Raman spectrum of pure oseltamivir phosphate API. The Raman spectrum of the capsules is the average of the Raman spectra for the different possible orientations for each capsule, as described in the preceding section. The Raman spectra of all the authentic capsules have a similar spectral profile that is dominated by fluorescence background. Weak Raman features below $700 \mathrm{~cm}^{-1}$ are present for all capsule spectra and are better resolved for the higher dosage strengths, 45 and $75 \mathrm{mg}$, as shown in Figure 1(a). This is in part due to the increasing capsule size and API loading which collectively allow for more reliable analysis when using the defocused $3 \mathrm{~mm}$ laser spot. The Raman spectrum of oseltamivir phosphate was acquired through the bottom of a 4 $\mathrm{mL}$ glass vial which gives rise to glass fluorescence signal centered around $\sim 1500 \mathrm{~cm}^{-1}$. Baseline subtraction according to the Lieber, et al. method [11] was applied to the average Raman spectra of the three dosage strengths and oseltamivir phosphate to facilitate comparison of the common Raman features between the oseltamivir phosphate and the capsule-form products, shown in Figure 1(b). Comparison of the baseline-subtracted data clearly shows that the bands at 870,1454 and $1653 \mathrm{~cm}^{-1}$ are due to oseltamivir phosphate peaks present in the capsule composite. The three prominent bands, that are present in the capsule spectra but absent in the oseltamivir phosphate spectrum, at $\sim 390,510$, and $630 \mathrm{~cm}^{-1}$ are likely due to titanium dioxide [12] which is an inactive ingredient present in the capsule formulation. The presence of both active and inactive ingredient bands in the capsule spectra reveals that the Raman method using the $3 \mathrm{~mm}$ spot size is able to probe the capsule shell to analyze the internal contents of the capsules.

A three component PCA model, shown in Figure 2, was created for exploratory purposes based on the spectra of 12 capsules from each of the three dosage strengths (36 total spectra). The calibration spectra for each capsule strength were each assigned to a different class according to dosage strength and the validation spectra were all left unassigned. The three dimensional scores plot in Figure 2 shows that the method is able to adequately distinguish between the dosage strengths, as designated by different ellipsoids. Although we noticed that the capsule
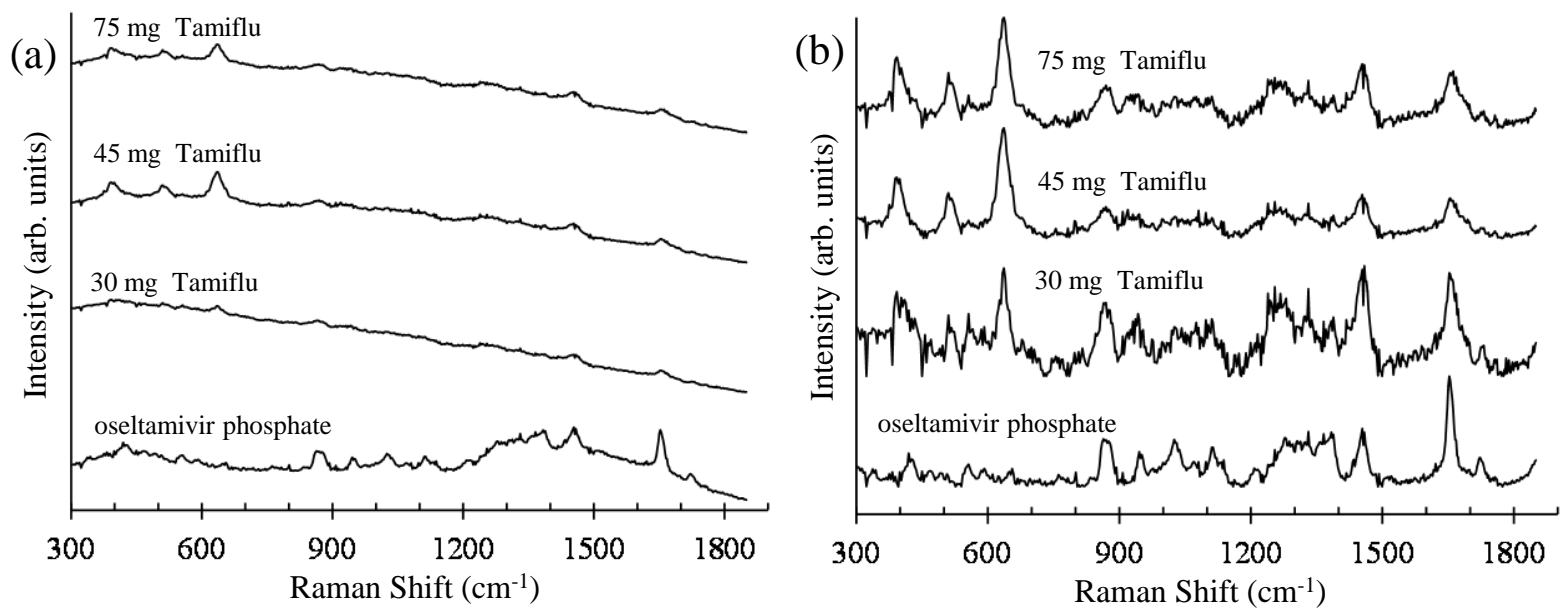

Figure 1. (a) Comparison of the average Raman spectra for each capsule strength with the active ingredient (oseltamivir phosphate) of Tamiflu; (b) Raman spectra were treated with baseline subtraction [11] and normalized to their maximum intensity over the full spectral range. 


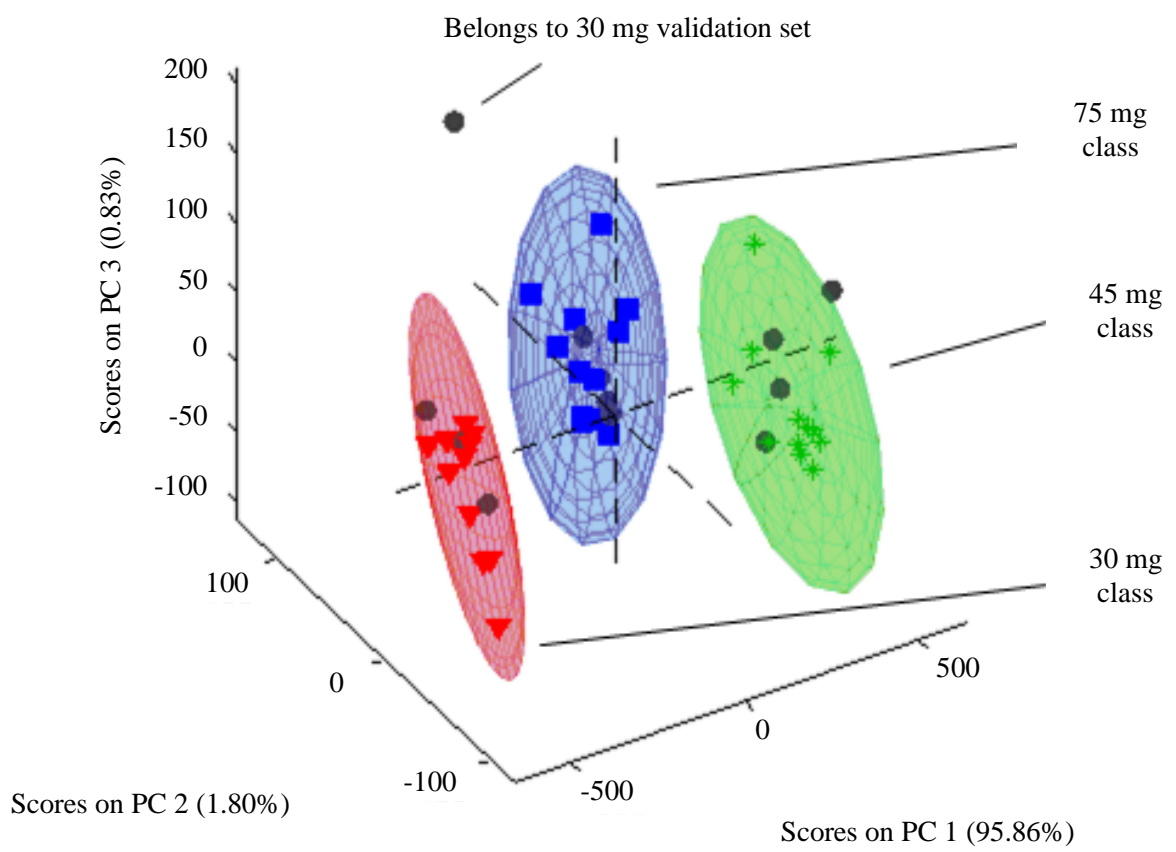

Figure 2. Evaluation of calibration and validation sets applied to PCA model of all dosages. The scores plot contains the three dosage classes in the calibration set (red, blue, green), as well as the validation samples (black). The $95 \%$ confidence limit is designated by an ellipsoid around each dosage class.

shell halves (being different colors, white or yellow) differently attenuate the Raman signal, the spectra shown in Figure 1 containing noticeable contribution from the capsule contents allow for sufficient discriminating characteristics for PCA to successfully differentiate between the three dosages in the calibration set. The validation samples, all authentic Tamiflu capsules in this case and shown as black circles in Figure 2, mostly align well within the $95 \%$ confidence limits for the score distribution for each strength, except for one sample of 30 mg dosage. A closer examination of this lone sample revealed that the Raman spectrum for that sample has lower intensity for API peaks (particularly at $1653 \mathrm{~cm}^{-1}$ ) and overall lower signal-to-noise across the spectrum, which caused it to lie outside the score distribution of its assigned class.

The same three principal component PCA model was used to predict the results for simulated counterfeit samples prepared using the capsule shells of authentic Tamiflu samples. Care was taken to refill the capsules such that the resulting masses were similar to bona fide capsules.

The counterfeit samples appeared to be essentially the same to the unaided eye as the authentic Tamiflu samples, as shown by the empty and starch-refilled counterfeit samples depicted in Figure 3(a), Figure 3(b). Acetaminophen, the active ingredient in Tylenol, has been reported as a commonly incorporated cheaper substitute present in counterfeits because of its ability to reduce fever and pain, leading the patient to believe that the medicine is producing a therapeutic effect [13]. Acetaminophen and other common, relatively inexpensive inactive ingredients were used to make the simulated counterfeits in this study. The inactive ingredients chosen were meant to be similar to those listed as ingredients in authentic Tamiflu samples [14]. The simulated counterfeits consisted of Tamiflu capsules that have been refilled with starch, titanium dioxide, starch/acetaminophen, titanium dioxide/acetaminophen, and talc/acetaminophen mixtures as well as empty Tamiflu capsules and capsuleform amantadine and acyclovir. A full roster of simulated counterfeits is listed in Table 1.

When analyzed within the three principal component PCA model containing all three strengths, 19 of the 20 simulated counterfeits were distinguished from authentic Tamiflu samples. Visual representation of the results is shown in Figure 3(c), which shows that the black circles corresponding to these simulated counterfeit samples are outside of the ellipsoids for the three dosage classes of calibration set. The region of the PCA model around the green ellipsoid for the $45 \mathrm{mg}$ data is expanded in Figure 3(d), illustrating the locations of the simulated counterfeit samples. Only one simulated counterfeit containing 51\%/49\% talc/acetaminophen binary mixture was incorrectly predicted to lie within the $95 \%$ confidence limit for $45 \mathrm{mg}$ dosage. 


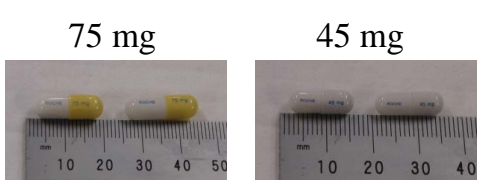

$30 \mathrm{mg}$

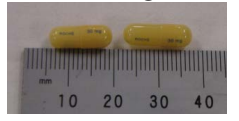

(a)

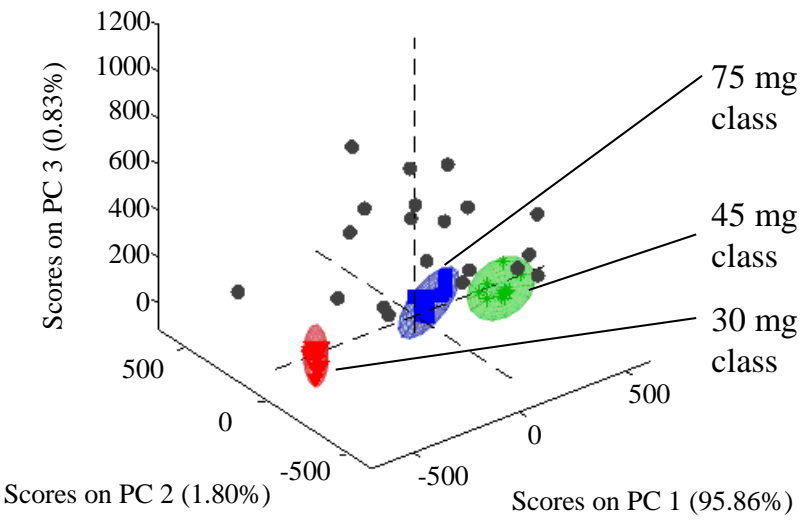

(c)

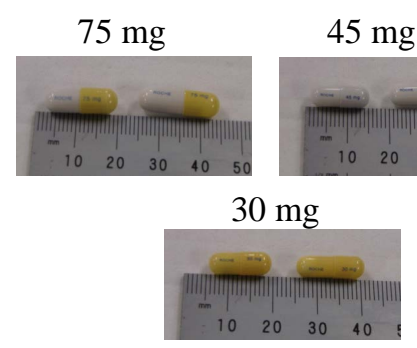

(b)

45 mg capsule of talc/acetaminophen

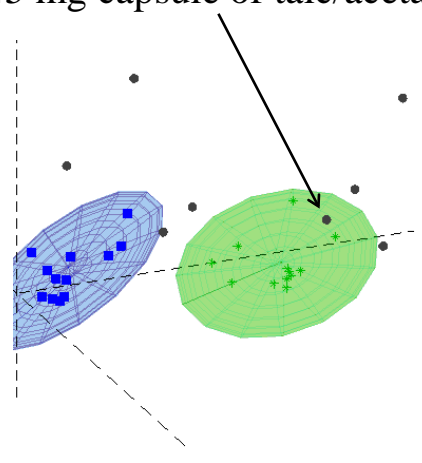

(d)

Figure 3. Simulated counterfeits and other capsule-form drug products were used to test the models. (a) Empty Tamiflu capsules, on right in each picture, are compared to authentic Tamiflu capsules; (b) Starch-refilled Tamiflu capsules, on right in each picture, are compared to authentic Tamiflu capsules; (c) PCA model constructed using all dosages is tested with simulated counterfeits and other capsule-form drug products. The calibration samples of $30 \mathrm{mg}$ (red), $45 \mathrm{mg}$ (green), and 75 mg (blue) are depicted, with 95\% confidence limits (shaded ellipsoids). The simulated counterfeits and other drug products are designated as black circles; (d) An elarged region of PCA model in (c) showing one simulated counterfeit sample predicted slightly inside the space for $45 \mathrm{mg}$ capsule of the calibration set.

Following exploratory PCA analysis, we applied the SIMCA classification algorithm to the data to determine the ability of the models to make predictions for both authentic Tamiflu samples from different batches as well as the simulated counterfeits. The SIMCA model was a collection comprised of three individual PCA models, one model for each capsule strength. The number of PCs and preprocessing for each capsule strength was chosen independently from the other two dosages. Thus the $30 \mathrm{mg}$ dosage model contained three PCs, while the 45 $\mathrm{mg}$ and $75 \mathrm{mg}$ dosage models contain two PCs. Each individual PCA model that comprises the SIMCA model is shown in Figure 4. The results shown in Figure 4 are similar to those shown in Figure 2 for the three principal components containing all the capsule strengths. The validation samples for each dosage were within the 95\% confidence limits for their respective models, except for the one $30 \mathrm{mg}$ sample. This sample also was predicted to lie outside the $30 \mathrm{mg}$ distribution for the PCA model comprised of the three dosage strengths (shown in Figure 2) and its unassigned prediction is likely due to the overall lower signal-to-noise observed in the Raman spectrum of the sample. Probabilities of the validation samples to be in each dosage class are listed in Table 1 . The last column shows the class predictions which were determined based on the probability that the sample is in any class. A test sample is assigned to a class if its probability is greater than 0.5 . As mentioned in the previous section, classes are unassigned for validation samples that have probabilities exceeding 0.5 for multiple classes or if there is no particular class where the 0.5 probability is exceeded. Except for the second $30 \mathrm{mg}$ sample, all validation samples were assigned probabilities exceeding the 0.5 threshold of being in their correct class and zero probability for any other dosage class. Thus, $92 \%$ of the validation samples from authentic Tamiflu were correctly classified as belonging to their respective dosage class. The SIMCA model was also able to correctly assign $100 \%$ of the simulated counterfeits and other capsule-form drug products as not belonging to any of the three dosage classes for Tamiflu, demonstrating robustness of the classification model. Even the 51\%/49\% talc/acetaminophen binary mixture, which was predicted to lie within the $95 \%$ confidence limit for $45 \mathrm{mg}$ dosage using PCA, was correctly identified as not belonging to any of the authentic Tamiflu classes. 
Table 1. Classification results from SIMCA model for the validation set and simulated counterfeits.

\begin{tabular}{|c|c|c|c|c|}
\hline Validation sample & $\begin{array}{l}\text { Probability in } \\
30 \text { mg class }\end{array}$ & $\begin{array}{l}\text { Probability in } \\
45 \text { mg class }\end{array}$ & $\begin{array}{l}\text { Probability in } \\
75 \text { mg class }\end{array}$ & $\begin{array}{c}\text { Class } \\
\text { prediction }\end{array}$ \\
\hline $30 \mathrm{mg}-1$ & 0.572 & 0 & 0 & $30 \mathrm{mg}$ class \\
\hline $30 \mathrm{mg}-2$ & 0 & 0 & 0 & none \\
\hline $30 \mathrm{mg}-3$ & 1 & 0 & 0 & 30 mg class \\
\hline $30 \mathrm{mg}-4$ & 1 & 0 & 0 & 30 mg class \\
\hline $45 \mathrm{mg}-1$ & 0 & 1 & 0 & $45 \mathrm{mg}$ class \\
\hline $45 \mathrm{mg}-2$ & 0 & 0.641 & 0 & 45 mg class \\
\hline $45 \mathrm{mg}-3$ & 0 & 0.715 & 0 & $45 \mathrm{mg}$ class \\
\hline $45 \mathrm{mg}-4$ & 0 & 1 & 0 & $45 \mathrm{mg}$ class \\
\hline $75 \mathrm{mg}-1$ & 0 & 0 & 1 & $75 \mathrm{mg}$ class \\
\hline $75 \mathrm{mg}-2$ & 0 & 0 & 1 & 75 mg class \\
\hline $75 \mathrm{mg}-3$ & 0 & 0 & 1 & 75 mg class \\
\hline $75 \mathrm{mg}-4$ & 0 & 0 & 1 & $75 \mathrm{mg}$ class \\
\hline \multicolumn{5}{|c|}{ Simulated counterfeits } \\
\hline 30 mg-empty capsule & 0 & 0 & 0 & None \\
\hline $30 \mathrm{mg}-100 \%$ starch & 0 & 0 & 0 & None \\
\hline $30 \mathrm{mg}-100 \% \mathrm{TiO}_{2}$ & 0 & 0 & 0 & None \\
\hline $30 \mathrm{mg}-50 \% \mathrm{starch} / 50 \%$ acetaminophen & 0 & 0 & 0 & None \\
\hline $30 \mathrm{mg}-51 \% \mathrm{TiO}_{2} / 49 \%$ acetaminophen & 0 & 0 & 0 & None \\
\hline $30 \mathrm{mg} \_51 \%$ talc/49\% acetaminophen & 0 & 0 & 0 & None \\
\hline 45 mg-empty capsule & 0 & 0 & 0 & None \\
\hline $45 \mathrm{mg}-100 \%$ starch & 0 & 0 & 0 & None \\
\hline $45 \mathrm{mg}-100 \% \mathrm{TiO}_{2}$ & 0 & 0 & 0 & None \\
\hline $45 \mathrm{mg}-50 \% \mathrm{starch} / 50 \%$ acetaminophen & 0 & 0 & 0 & None \\
\hline $45 \mathrm{mg}-51 \% \mathrm{TiO}_{2} / 49 \%$ acetaminophen & 0 & 0 & 0 & None \\
\hline $45 \mathrm{mg}-51 \%$ talc/49\% acetaminophen & 0 & 0 & 0 & None \\
\hline 75 mg_empty capsule & 0 & 0 & 0 & None \\
\hline $75 \mathrm{mg}-100 \%$ starch & 0 & 0 & 0 & None \\
\hline $75 \mathrm{mg}-100 \% \mathrm{TiO}_{2}$ & 0 & 0 & 0 & None \\
\hline $75 \mathrm{mg}-50 \%$ starch $/ 50 \%$ acetaminophen & 0 & 0 & 0 & None \\
\hline $75 \mathrm{mg}-51 \% \mathrm{TiO}_{2} / 49 \%$ acetaminophen & 0 & 0 & 0 & None \\
\hline $75 \mathrm{mg}-51 \%$ talc/49\% acetaminophen & 0 & 0 & 0 & None \\
\hline Capsule containing acyclovir & 0 & 0 & 0 & None \\
\hline Capsule containing amantadine & 0 & 0 & 0 & None \\
\hline
\end{tabular}




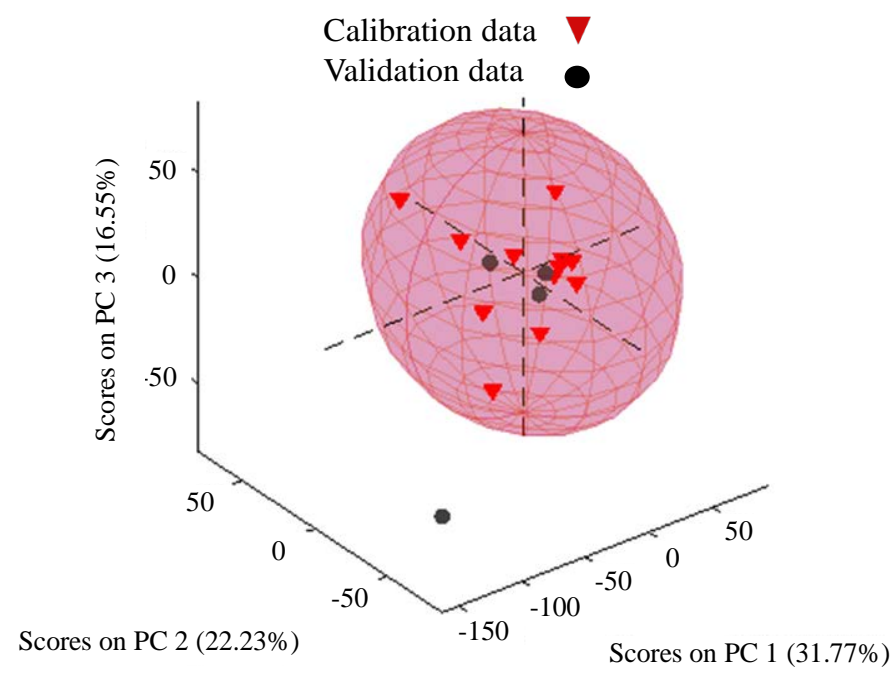

(a)

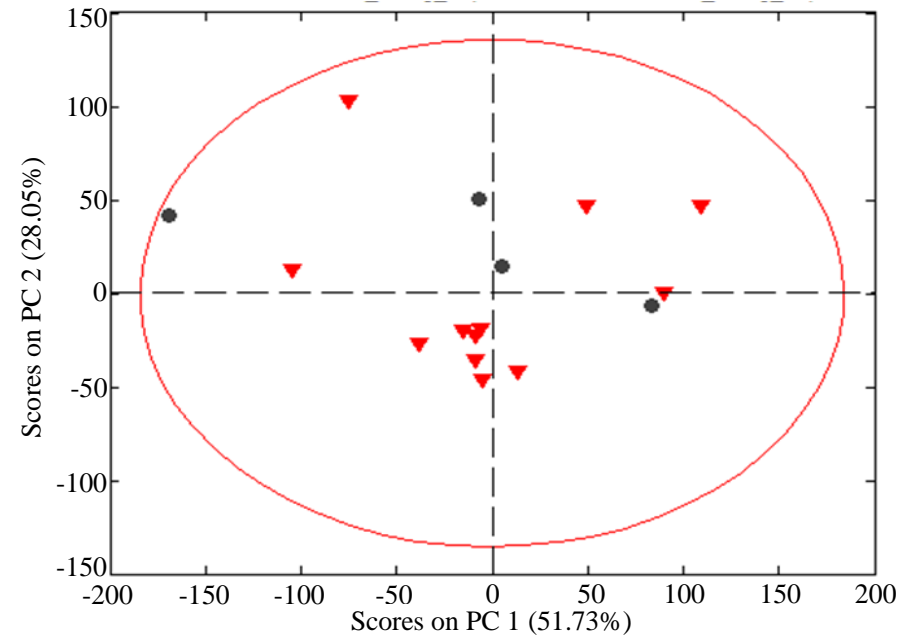

(b)

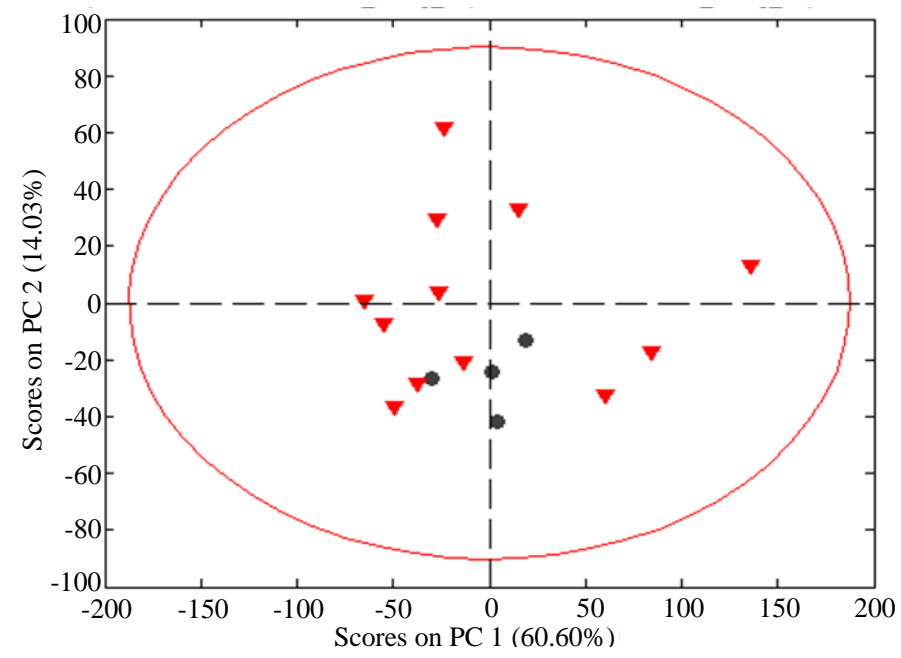

(c)

Figure 4. Individual PCA models for each dosage: (a) $30 \mathrm{mg}$, (b) $45 \mathrm{mg}$, and (c) $75 \mathrm{mg}$, with calibration and validation data shown. 95\% confidence limits for these models are denoted by the red sphere (a) and red circles (b) (c). 


\section{Conclusion}

This study has the potential to transform the way medicines are tested by facilitating high throughput screening of samples before reaching consumers. Currently products typically undergo laboratory testing to meet regulatory product specifications. These tests typically rely on laboratory-based tests for assay, dissolution and impurities. These tests cannot be carried out in the field and, since they require many analyst hours to complete, limit the number of individual samples that can be tested. The results presented here show that portable Raman methods coupled with classification algorithms may be used to identify finished medicines in the field in a non-destructive fashion. By preserving the intact capsule, these methods make it possible to conduct further testing, should it be necessary, to confirm field results by laboratory testing. The classification methods were able to screen through the capsule shell and identify simulated counterfeit samples and those from different capsule-form drugs.

\section{Acknowledgements}

This project was supported in part by an appointment (Y.L.L.) to the Research Participation Program at the Center for Drug Evaluation and Research administered by the Oak Ridge Institute for Science and Education through an interagency agreement between the US Department of Energy and the US Food and Drug Administration. Support for this project was received through the CDER Critical Path Program and the FDA Medical Counter-measures Initiative (MCMi-challenge).

\section{Disclaimer}

This article reflects the views of the authors and should not be construed to represent FDA's views or policies.

\section{References}

[1] Patel, A. and Gorman, S.E. (2009) Stockpiling Antiviral Drugs for the Next Influenza Pandemic. Clinical Pharmacology Therapeutics, 86, 241-243. http://dx.doi.org/10.1038/clpt.2009.142

[2] Degardin, K., Roggo, Y. and Margot, P. (2014) Understanding and Fighting the Medicine Counterfeit Market. Journal of Pharmaceutical and Biomedical Analysis, 87, 167-175. http://dx.doi.org/10.1016/j.jpba.2013.01.009

[3] Emergency Use Authorization (EUA) Review Oseltamivir Phosphate for Swine Influenza A. http://www.fda.gov/downloads/Drugs/DrugSafety/InformationbyDrugClass/UCM153547.pdf

[4] Stockpiled Antivirals at or Nearing Expiration. http://www.fda.gov/NewsEvents/PublicHealthFocus/ucm154962.htm

[5] FDA Warns of Counterfeit Tamiflu Product. http://www.empr.com/fda-warns-of-counterfeit-tamiflu-product/article/172765

[6] Rodriguez, J.D., Gryniewicz-Ruzicka, C.M., Arzhantsev, S., Kauffman, J.F. and Buhse, L.F. (2014) Rapid Screening Methods for Pharmaceutical Surveillance. Science and the Law: Analytical Data in Support of Regulation in Health, Food, and the Environment, American Chemical Society, 149-168.

[7] de Peinder, P., Vredenbregt, M.J., Visser, T. and de Kaste, D. (2008) Detection of Lipitor ${ }^{\circledR}$ Counterfeits: A Comparison of NIR and Raman Spectroscopy in Combination with Chemometrics. Journal of Pharmaceutical and Biomedical Analysis, 47, 688-694. http://dx.doi.org/10.1016/j.jpba.2008.02.016

[8] Degardin, K., Roggo, Y., Been, F. and Margot, P. (2011) Detection and Chemical Profiling of Medicine Counterfeits by Raman Spectroscopy and Chemometrics. Analytica Chimica Acta, 705, 334-341. http://dx.doi.org/10.1016/j.aca.2011.07.043

[9] Hargreaves, M.D., Macleod, N.A., Smith, M.R., Andrews, D., Hammond, S.V. and Matousek, P. (2011) Characterisation of Transmission Raman Spectroscopy for Rapid Quantitative Analysis of Intact Multi-Component Pharmaceutical Capsules. Journal of Pharmaceutical and Biomedical Analysis, 54, 463-468. http://dx.doi.org/10.1016/i.jpba.2010.09.015

[10] Eigenvector Wiki: Sample Classification Predictions. http://wiki.eigenvector.com/index.php?title=Sample_Classification_Predictions

[11] Lieber, C.A. and Mahadevan-Jansen, A. (2003) Automated Method for Subtraction of Fluorescence from Biological Raman Spectra. Applied Spectroscopy, 57, 1363-1367. http://dx.doi.org/10.1366/000370203322554518

[12] Balachandran, U. and Eror, N.G. (1982) Raman Spectra of Titanium Dioxide. Journal of Solid State Chemistry, 42, 276-282. http://dx.doi.org/10.1016/0022-4596(82)90006-8 
[13] Weaver, A., Reiser, H., Bartis, T., Benvenuti, M., Ghosh, D., Hunckler, M., Joy, B., Koenig, L., Raddell, K. and Lierberman, M. (2013) Paper Analytical Devices for Fast Field Screening of Beta Lactam Antibiotics and Anti-Tuberculosis Pharmaceuticals. Analytical Chemistry, 85, 6453-6460. http://dx.doi.org/10.1021/ac400989p

[14] EMC+ Online Database Tamiflu 75 mg Capsule. http://www.medicines.org.uk/emcmobile/medicine/10446/spc\#COMPOSITION 